\title{
Ovarian Microcystic Stromal Tumor
}

National Cancer Institute

\section{Source}

National Cancer Institute. Ovarian Microcystic Stromal Tumor. NCI Thesaurus. Code C121953.

A benign, unilateral tumor that arises from the ovary and is characterized by the presence of conspicuous microcystic changes, cellular areas, and a fibrous stroma. 